\title{
THE FUNDAMENTAL SOLUTION IN AXIALLY SYMMETRIC POTENTIAL THEORY
}

\author{
By ALBERT E. HEINS $\dagger$
}

[Received 8 October 1973]

\section{Introduction}

We propose to discuss here the equivalence between the standard free space Green's function in axially symmetric potential theory and several others which have been used in the past decade ([1], [2], and [3]). The interest in these forms is due to the fact that it is possible to produce integral equations of the Volterra type to formulate boundary-value problems rather than equations of the Fredholm type which arise in the classical case and this, of course, is an advantage which we cannot afford to overlook. Incidentally, it is from these latter forms of the Green's functions that we can derive numerous representations which have appeared in the literature since the work of Laplace.

We recall that the partial differential equation

$$
\frac{\partial^{2} \varphi}{\partial x^{2}}+\frac{\partial^{2} \varphi}{\partial y^{2}}+\frac{\partial^{2} \varphi}{\partial z^{2}}=0
$$

has the fundamental solution $\left[(x-\alpha)^{2}+(y-\beta)^{2}+(z-\gamma)^{2}\right]^{-\frac{1}{2}} / 4 \pi$ and the integral, with $x=r \cos \theta, y=r \sin \theta, \alpha=t \cos \theta^{\prime}, \beta=t \sin \theta^{\prime}$,

$$
\frac{1}{4 \pi} \int_{0}^{2 \pi} \frac{d \theta^{\prime}}{\left[(x-\alpha)^{2}+(y-\beta)^{2}+(z-\gamma)\right]^{\frac{1}{2}}}
$$

is independent of $\theta$. Therefore (1.1) is actually a solution of the elliptic partial differential equations

$$
\frac{\partial^{2} \varphi}{\partial r^{2}}+\frac{1}{r} \frac{\partial \varphi}{\partial r}+\frac{\partial^{2} \varphi}{\partial z^{2}}=0
$$

or

$$
\frac{\partial^{2} \varphi}{\partial t^{2}}+\frac{1}{t} \frac{\partial^{2} \varphi}{\partial t^{2}}+\frac{\partial^{2} \varphi}{\partial \gamma^{2}}=0
$$

The $n$th harmonic of the fundamental solution is either

$$
\begin{aligned}
\frac{1}{4 \pi} \int_{0}^{2 \pi} \frac{\cos n \theta^{\prime} d \theta^{\prime}}{\left[(z-\gamma)^{2}+r^{2}+t^{2}-2 \operatorname{tr} \cos \left(\theta-\theta^{\prime}\right)\right]^{\frac{1}{2}}} \\
=\frac{1}{2 \pi} \int_{0}^{\pi} \frac{\cos n \theta \cos n \theta^{\prime \prime} d \theta^{\prime \prime}}{\left[(z-\gamma)^{2}+r^{2}+t^{2}-2 t r \cos \theta^{\prime \prime}\right]^{\frac{1}{2}}}
\end{aligned}
$$

† This research was supported in part by the Air Force Office of Scientific Research, Office of Aerospace Research, USAF, under AFOSR Grant No. 71-2081.

Proc. London Math. Soc. (3) 29 (1974) 735-749 
or

$$
\begin{aligned}
& \frac{1}{4 \pi} \int_{0}^{2 \pi} \frac{\sin n \theta^{\prime} d \theta^{\prime}}{\left[(z-\gamma)^{2}+r^{2}+t^{2}-2 t r \cos \left(\theta-\theta^{\prime}\right)\right]^{\frac{1}{3}}} \\
&=\frac{1}{2 \pi} \int_{0}^{\pi} \frac{\sin n \theta \cos n \theta^{\prime \prime} d \theta^{\prime \prime}}{\left[(z-\gamma)^{2}+r^{2}+t^{2}-2 t r \cos \theta^{\prime \prime}\right]^{\frac{1}{t}}} .
\end{aligned}
$$

Observe that, save for the factors $\sin n \theta$ and $\cos n \theta,(1.3)$ and (1.4) are the same. It is clear that since (1.3) and (1.4) satisfy the equation

$$
\frac{\partial^{2} \varphi}{\partial r^{2}}+\frac{1}{r} \frac{\partial \varphi}{\partial r}-\frac{n^{2}}{r^{2}} \varphi+\frac{\partial^{2} \varphi}{\partial z^{2}}=0
$$

we can rewrite (1.5) by the substitution $r^{n} A(r, z)=\varphi(r, z)$ as

$$
\frac{\partial^{2} A}{\partial r^{2}}+\frac{1+2 n}{r} \frac{\partial A}{\partial r}+\frac{\partial^{2} A}{\partial z^{2}}=0
$$

When $n$ is a positive integer, we may characterize equation (1.6) in another fashion. Let us examine Laplace's equation in $m$ dimensions, that is,

$$
\sum_{i=1}^{m} \frac{\partial^{2} \varphi}{\partial x_{i}^{2}}=0
$$

and write the coordinates $x_{i}, i=1, \ldots, m$, in terms of 'cylindrical coordinates' in $m$ dimensions. That is, we put

$$
\begin{aligned}
x_{1}= & r \sin \theta_{1} \ldots \sin \theta_{m-2} \\
x_{2} & =r \sin \theta_{1} \ldots \cos \theta_{m-2} \\
\cdot & \cdot \cdot \cdot \cdot \cdot \cdot \cdot \\
x_{m-1} & =r \cos \theta_{1} \\
x_{m} & =x_{m} .
\end{aligned}
$$

Solutions of (1.7) which are independent of the angles $\theta_{i}, i=1, \ldots, m-2$, satisfy the equation

$$
\frac{\partial^{2} \varphi}{\partial x_{m}^{2}}+\frac{\partial^{2} \varphi}{\partial r^{2}}+\frac{m-2}{r} \frac{\partial \varphi}{\partial r}=0
$$

where $r^{2}=\sum_{i=1}^{m-1} x_{i}{ }^{2}$, and thus when $m-2=2 n+1$ there is a correspondence between the amplitude of the $n$th harmonic of Laplace's equation in three dimensions and an angularly independent solution in $m$-dimensional cylindrical coordinates.

In order to display this correspondence, we recall that the spherically symmetric form of equation (1.7) is

$$
\frac{\partial^{2} \varphi}{\partial \rho^{2}}+\frac{m-1}{\rho} \frac{\partial \varphi}{\partial \rho}=0
$$


where $\rho^{2}=\sum_{i=1}^{m} x_{i}{ }^{2}$ and this differential equation has the singular solution $\rho^{2-m}$. But if $\rho^{2-m}$ is a solution of equation (1.7), so is $\rho_{1}{ }^{2-m}=\left[\sum_{i=1}^{m}\left(x_{i}-\xi_{i}\right)^{2}\right]^{1-\frac{1}{\mathrm{j}} m}$. We shall therefore put

$$
\rho_{1}^{2}=\left(x_{m}-\xi_{m}\right)^{2}+r^{2}+b^{2}-2 r b \cos \theta_{1},
$$

where now $b^{2}=\sum_{i=1}^{m-1} \xi_{i}{ }^{2}$ and the angle $\theta_{1}$ is taken to be the angle between the vectors $\bar{r}$ and $\bar{b}$ (that is, we measure $\theta_{1}$ in the cylindrical coordinate transformation with respect to the vector $\bar{b}$ ). Hence a fundamental solution of equation (1.8) which is independent of the angle $\theta_{i}$, $i=1, \ldots, m-2$, is given by the integral

$$
\int_{\partial \Omega_{m-1}} \rho_{1}{ }^{2-m} d \Omega,
$$

where $\partial \Omega_{m-1}$ is the boundary of the unit sphere in $m-1$ dimensions with centre at $\left(\xi_{1}, \ldots, \xi_{m-1}\right)$.

We normalize this fundamental solution in the same manner as we do for the case when $m=3$. We require that the 'flow' from the point $\left(\xi_{1}, \ldots, \xi_{m}\right)$ over a unit sphere be unity, that is,

$$
C(2-m) \int_{\partial \Omega_{m}} \rho_{1}{ }^{1-m} d \Omega=-1
$$

and hence ([4]), $C=\Gamma\left(\frac{1}{2} m-1\right) /\left(4 \pi^{\frac{1 m}{m}}\right)$. With this normalizing factor, the axially symmetric fundamental solution in $m$ dimensions, $m=3,4, \ldots$, is

$$
\begin{aligned}
& \frac{\Gamma\left(\frac{1}{2} m-1\right)}{4 \pi^{\frac{1}{b}}} \int_{0}^{\pi} \frac{\sin ^{m-3} \theta_{1} d \theta_{1}}{\left[\left(x_{m}-\xi_{m}\right)^{2}+r^{2}+b^{2}-2 r b \cos \theta_{1}\right]^{\frac{13 m-1}{3}}} \\
& \times \int_{0}^{2 \pi} \ldots \int_{0}^{2 \pi} \sin ^{m-4} \theta_{2} \ldots \sin \theta_{m-2} d \theta_{2} \ldots d \theta_{m-2}
\end{aligned}
$$

$$
=\frac{1}{2 \pi} \int_{0}^{\pi} \frac{\sin ^{m-3} \theta_{1} d \theta_{1}}{\left[\left(x_{m}-\xi_{m}\right)^{2}+r^{2}+b^{2}-2 r b \cos \theta_{1}\right]^{]^{m-1}}} \text {. }
$$

We now replace $x_{m}$ by $z$ and $\xi_{m}$ by $\gamma$ to conform with our previous notation and discuss the equivalence of (1.10) and

$$
\frac{1}{2 \pi(r b)^{n}} \int_{0}^{\pi} \frac{\cos n \theta d \theta}{\left[(z-\gamma)^{2}+r^{2}+b^{2}-2 r b \cos \theta\right]^{\frac{1}{2}}}
$$

for the case when $m$ is an odd integer, which is greater than or equal to 3, and $m-3=2 n$. The presence of the factor $r b$ is due to the fact that (1.11) is required to be symmetric in $r$ and $b$ and is to satisfy equation (1.6) as a function of $r$ and $z$ as well as (1.6) with $z$ replaced by $\gamma$ and $r$ by $b$. Let us first observe that both (1.10) and (1.11) have the behaviour

$$
\frac{1.3 \ldots(2 n-1)}{2^{n+1} n ! R^{2 n+1}}+\frac{z \gamma(1.3 \ldots(2 n+1))}{2^{n+2} n ! R^{2 n+3}}+O\left(\frac{1}{R^{2 n+3}}\right),
$$


when $R \rightarrow \infty$, where $R^{2}=z^{2}+r^{2}$. It is also possible to calculate the $R$ derivative from these integrals. Now let $\varepsilon^{2}=(z-\gamma)^{2}+(r-b)^{2}, b>0$. Then for $\varepsilon \rightarrow 0$, the integrals in (1.10) and (1.11) have the same leading terms $-\ln \varepsilon / 2 \pi b^{2 n+1}+O(\varepsilon \ln \varepsilon)$. We obtain this behaviour for (1.10) by observing that it is an abelian integral which reduces to a hypergeometric function. For (1.11), we expand $\cos n \theta$ in powers of $\sin ^{2} \frac{1}{2} \theta$ and note that the constant term is an elliptic integral which possesses the above logarithmic behaviour for $\varepsilon \rightarrow 0$ as well as the same type of error. It is possible to calculate the $\varepsilon$-derivative for $\varepsilon \rightarrow 0$. Finally, we note that (1.10) and (1.11) are even in $r$ and $b$ and indeed have vanishing $r$-derivatives at $r=0$.

We now claim that (1.10) and (1.11) are the same. They both behave as $O\left(R^{-2 n-3}\right)$ when $R \rightarrow \infty$, have a vanishing $r$ derivative on $r=0$, and their difference is $O(\varepsilon \ln \varepsilon)$ when $\varepsilon \rightarrow 0$. Then if we put (1.10) equal to $B(r, z ; b, \gamma)$ and $(1.11)$ equal to $C(r, z ; b, \gamma)$, their difference $D(r, z ; b, \gamma)$ obeys the integral relation (which we derive directly from the divergence theorem)

$$
\begin{aligned}
\iint\left[r^{2 n+1} \frac{\partial^{2} D}{\partial z^{2}}+\frac{\partial}{\partial r} r^{2 n+1} \frac{\partial D}{\partial r}+r^{2 n+1}\left\{\left(\frac{\partial D}{\partial r}\right)^{2}+\left(\frac{\partial D}{\partial z}\right)^{2}\right\}\right] & \rho d \rho d \theta \\
& =-\int_{0}^{\pi} r^{2 n+1} R D \frac{\partial D}{\partial R} d \theta,
\end{aligned}
$$

where $z=\rho \cos \theta, r=\rho \sin \theta$, and the area integral is carried out over the semi-circle $0 \leqslant \rho \leqslant R, 0 \leqslant \theta \leqslant \pi$. Now the $\theta$-integral is $O(1 / R)$ when $R \rightarrow \infty$ and the sum of the first two terms in the area integral vanishes in view of the fact that $D$ satisfies equation (1.6). There are no difficulties in the neighbourhood of $r=b$ and $z=\gamma$. Hence we have for $R \rightarrow \infty$, that is, for $0 \leqslant \rho, 0 \leqslant \theta \leqslant \pi$,

$$
\iint r^{2 n+1}\left[\left(\frac{\partial D}{\partial r}\right)^{2}+\left(\frac{\partial D}{\partial z}\right)^{2}\right] \rho d \rho d \theta=0
$$

and therefore $D$ is a constant. However, we can show that $B=C$ at $r=0$ and therefore $D$ vanishes identically and hence $B=C$.

Let us observe that if $m$ is a positive number which exceeds $2,(1.10)$ is still a solution of equation (1.8). We may therefore speak of (1.10) as an axially symmetric fundamental solution in the 'meridian plane $(z, r)$ of a fictitious space of $m$ dimensions'. Our present efforts, however, will be devoted only to the cases $m=3,5, \ldots$.

In $\S 2$, we show that (1.10) can be converted to an abelian integral from which we shall derive other useful forms, in particular, one which is attributed to Laplace and one which was used by Carleman in the case 
when $m=3$. We may remark, parenthetically, that (1.10) may also be expressed in terms of an improper integral containing Bessel functions of order $\frac{1}{2}(m-3)$, as we can infer from an integral due to Lipschitz ([5]), but this form of the fundamental solution has led to many complications in the past. In $\S 3$, we shall show that we can derive Volterra integral equations for boundary-value problems of axially symmetric potential theory with the forms of the fundamental solution which we derive, while $\S 4$ will be devoted to two examples.

\section{The conversion of the fundamental solution}

We shall now show that it is possible to convert (1.11) into two other forms which will reveal their dependence on either of the complex variables $z+i r$ or $\gamma+i b$. The one which depends on $z+i r$ is the basis for the representation of Laplace, which may be derived by replacing Legendre functions by their integral representations in the expansion of a solution of equation (1.6). It is difficult to derive the properties of Laplace's representation in this manner. A representation similar to Laplace's was derived by Poisson ([6]) for the equation (1.6) when $z$ is replaced by $i z$ but the method assumes the analyticity of the solution. We shall presently see that these representations for the fundamental solution of equations (1.6) arise from a transformation of the abelian integral (1.10). These will be very useful in converting some of the Fredholm integral equations of axially-symmetric potential theory to Volterra equations.

Let us now observe that (1.10) may be written as

$$
\frac{2^{m-4}}{\pi(4 r b)^{\frac{b}{\sharp} m-1}} \int_{0}^{1} \frac{[t(1-t)]^{\frac{3}{m-2}} d t}{[Z-t]^{\frac{3}{m-1}}}
$$

where

$$
Z=\frac{(z-\gamma)^{2}+(r+b)^{2}}{4 r b} \geqslant 1
$$

if we put $t=\cos ^{2} \frac{1}{2} \theta$. Then we have an abelian integral which we may convert into several different forms with a bilinear transformation. We can map the upper or lower half of the complex $t$-plane into the interior of a circle in the $\lambda$-plane (with the real axis of the $t$-plane mapping into the boundary of a circle). Then with the transformation

$$
\lambda=\frac{A+B t}{C+D t},
$$

we take the points $(0,0),(1,0)$, and $(Z, 0)$ of the $t$-plane into points of the boundary of the circle in the $\lambda$-plane which we take to be symmetric with respect to the imaginary axis of the $\lambda$-plane. We first introduce parameters 
$\alpha$ (real) and $\sigma=\sigma_{1}+i \sigma_{2}$ which we shall presently choose for simplication. We take $t(0,0)$ into $\lambda(-\alpha), t(1,0)$ into $\lambda(\alpha), t(Z, 0)$ into $\lambda(\sigma)$, and $t(\infty)$ into $\lambda(-\bar{\sigma})$. Then we find that

$$
A=\frac{D}{2}(\alpha+\bar{\sigma}), \quad B=-\bar{\sigma} D, \quad C=-\frac{(\alpha+\bar{\sigma}) D}{2 \alpha},
$$

and

$$
Z=\frac{\left(\alpha+\sigma_{1}\right)^{2}+\sigma_{2}^{2}}{4 \alpha \sigma_{1}} .
$$

Upon using the transformation (2.1) in the abelian integral, we get

$$
\frac{1}{2 \pi} \frac{1}{\alpha^{\frac{1}{m-2}}}\left(\frac{\sigma_{1}}{r b}\right)^{\frac{1 m-1}{m}} \int_{-\alpha}^{\alpha} \frac{\left(\alpha^{2}-\lambda^{2}\right)^{\ddagger m-2}}{[(\sigma-\lambda)(\bar{\sigma}+\lambda)]^{]^{m-1}-1}} .
$$

Various forms may be derived from (2.2). To start, we put $\lambda=\alpha \cos \theta$ to obtain

$$
\frac{1}{2 \pi} \int_{0}^{\pi}\left(\frac{\sigma_{1} \alpha}{r b}\right)^{\frac{1}{m-1}} \frac{\sin ^{m-3} \theta d \theta}{\left[\sigma_{1}^{2}+\left(\sigma_{2}+i \alpha \cos \theta\right)^{2}\right]^{\frac{1 m-1}{m}}} .
$$

Now we notice that we can eliminate the factor $\sigma_{1} \alpha / r b$ if we choose either $\sigma_{1}=r, \alpha=b$ or $\sigma_{1}=b, \alpha=r$ and $\sigma_{2}=z-\gamma$. These choices lead to

$$
\frac{1}{2 \pi} \int_{0}^{\pi} \frac{\sin ^{m-3} \theta d \theta}{\left[r^{2}+(z-\gamma+i b \cos \theta)^{2}\right]^{t m-1}}
$$

and

$$
\frac{1}{2 \pi} \int_{0}^{\pi} \frac{\sin ^{m-3} \theta d \theta}{\left[b^{2}+(z-\gamma+i r \cos \theta)^{2}\right]^{+m-1}} .
$$

We note further that the $r$ - (or $b$-) derivative of (2.3) and (2.4) vanishes at $r=0$ or $b=0$. Therefore these integrals are two forms of the Green's function which satisfies the Neumann boundary condition on the line $r=0$ or $b=0$ for equation (1.8) in the half-plane $r>0$. Henceforth, we denote $(2.3)$ and $(2.4)$ by $G(z, r ; \gamma, b)$.

Associated with an axially symmetric potential function is an axially symmetric stream function $\psi(z, r)$, which is related to $\varphi(z, r)$ by the Beltrami-Stokes equations. That is,

$$
r^{m-2} \frac{\partial \varphi}{\partial z}=\frac{\partial \psi}{\partial r} \quad \text { and } \quad r^{m-2} \frac{\partial \varphi}{\partial r}=-\frac{\partial \psi}{\partial z} .
$$

$\psi$ satisfies the elliptic partial differential equation

$$
\frac{\partial^{2} \psi}{\partial z^{2}}+\frac{\partial^{2} \psi}{\partial r^{2}}-\frac{m-2}{r} \frac{\partial \psi}{\partial r}=0 \quad(m>2) .
$$

There are occasions when we shall be required to know the fundamental 
solution of equation (2.6). To this end, we employ a device due to Weinstein ([1]). We put $\psi=r^{m-1} A$ and find that $A$ satisfies an elliptic partial differential equation for an axially symmetric potential function, that is

$$
\frac{\partial^{2} A}{\partial z^{2}}+\frac{\partial^{2} A}{\partial r^{2}}+\frac{m}{r} \frac{\partial A}{\partial r}=0 .
$$

It follows from our remarks in $\S 1$ that the fundamental solution of equation $(2.6)$ is

$$
\begin{aligned}
& \frac{r^{m-1}}{2 \pi} \int_{0}^{\pi} \frac{\sin ^{m-1} \theta d \theta}{\left[(z-\gamma)^{2}+r^{2}+b^{2}-2 r b \cos \theta\right]^{\frac{3}{3}}} \\
& \quad=\frac{r^{m-1}}{2 \pi} \int_{0}^{\pi} \frac{\sin ^{m-1} \theta d \theta}{\left[r^{2}+(z-r+i b \cos \theta)^{2}\right]^{3 m}}, \text { and so on. }
\end{aligned}
$$

While the fundamental solution of equation (1.8) is $O\left(R^{2-m}\right)$ when $R=\left[z^{2}+r^{2}\right]^{\sharp} \rightarrow \infty$, the one associated with equation $(2.6)$ is $O\left(r^{m-1} / R^{m}\right)$ when $R \rightarrow \infty$. Furthermore, for $m>1$, the latter vanishes on the axis $r=0$ but it still possesses the logarithmic singularity in the neighbourhood of the point $z=\gamma, r=b$. Let us stress, in passing, that the stream function associated with the fundamental potential function is different from the fundamental solution associated with equation (2.6). For one thing, the former stream function does not possess a logarithmic singularity (and is actually 2-valued along the cut $z=\gamma, r>b)$. Its multivalued character has been emphasized by Weinstein on numerous occasions.

In the case when $m$ is an odd integer which is greater than or equal to 3, we observe that the denominator in (2.3), for example, may have its degree lowered by integration by parts. Suppose $m=5$, then (2.3) may be written as

$$
\frac{1}{2 \pi i b r^{2}} \int_{0}^{\pi} \frac{\cos \theta(z-\gamma+i b \cos \theta) d \theta}{\left[r^{2}+(z-\gamma+i b \cos \theta)^{2}\right]^{\frac{1}{2}}}
$$

and (2.4) may be written as

$$
\frac{1}{2 \pi i r b^{2}} \int_{0}^{\pi} \frac{\cos \theta(z-\gamma+i r \cos \theta) d \theta}{\left[b^{2}+(z-\gamma+i r \cos \theta)^{2}\right]^{\frac{1}{2}}} .
$$

Such forms will be useful in the following sections.

\section{Some integral equations in potential theory}

We recall that an exterior boundary-value problem for the Laplace equation

$$
\varphi_{x x}+\varphi_{\nu y}+\varphi_{z z}=0
$$


with a Dirichlet boundary condition $\varphi=0$ on $\partial S$, has the representation

$$
\begin{aligned}
\varphi(x, y, z)=a_{0}+a_{1} x+a_{2} y+a_{3} z \\
+\frac{1}{4 \pi} \iint_{\partial S}\left[\frac{\partial \varphi}{\partial n^{\prime}}\right] \frac{d S}{\left[\left(x-x^{\prime}\right)^{2}+\left(y-y^{\prime}\right)^{2}+\left(z-z^{\prime}\right)^{2}\right]^{\sharp}} .
\end{aligned}
$$

The symbol $\left[\partial \varphi / \partial n^{\prime}\right]$ is to be interpreted as either the discontinuity of the normal derivative of $\partial \varphi / \partial n$ on an open surface or merely $\partial \varphi / \partial n$ on a closed surface. The surface is a regular one in the sense of Kellogg ([7]). The term $a_{0}+a_{1} x+a_{2} y+a_{3} z$, where the $a_{i}$ are arbitrary constants, gives us the behaviour of $\varphi$ at infinity and is, of course, a solution of equation (3.1). Such a term gives us the possibility of discussing a constant potential as well as a uniform potential gradient at infinity, two common situations in potential theory.

Now we specialize $\partial S$ to be a surface of revolution about the $x$-axis, so that in terms of cylindrical coordinates $(x, r, \theta)$, where $y=r \cos \theta$, $z=r \sin \theta, 0 \leqslant \theta \leqslant 2 \pi, r>0,(3.2)$ becomes

$$
\begin{aligned}
\varphi(x, r \cos \theta, r \sin \theta)=a_{0}+a_{1} x+a_{2} r \cos \theta+a_{3} r \sin \theta \\
+\frac{1}{4 \pi} \iint_{\partial S}\left[\frac{\partial \varphi}{\partial n^{\prime}}\right] \frac{b d \theta^{\prime} d \sigma}{\left[(x-x)^{2}+r^{2}+b^{2}-2 r b \cos \left(\theta-\theta^{\prime}\right)\right]^{\frac{1}{t}}} .
\end{aligned}
$$

Here $y^{\prime}=b \cos \theta^{\prime}$ and $z^{\prime}=b \sin \theta^{\prime}, 0 \leqslant \theta^{\prime} \leqslant 2 \pi, b>0$, and $d \sigma$ is the element of arc-length in a meridian plane. We observe that because of the conditions at infinity which we have chosen, (3.3) may be written as three single representations. That is,

$$
\begin{aligned}
\frac{1}{2 \pi} \int_{0}^{2 \pi} \varphi(x, r \cos \theta, r \sin \theta) d \theta=a_{0}+a_{1} x \\
\quad+\frac{1}{8 \pi^{2}} \iint_{\partial S} b\left[\frac{\partial \varphi}{\partial n^{\prime}}\right] d \theta^{\prime} d \sigma \int_{0}^{2 \pi} \frac{d \theta}{\left[\left(x-x^{\prime}\right)^{2}+r^{2}+b^{2}-2 r b \cos \left(\theta-\theta^{\prime}\right)\right]^{\frac{3}{2}}} .
\end{aligned}
$$

Upon putting

$$
\varphi^{0}(x, r)=\frac{1}{2 \pi} \int_{0}^{2 \pi} \varphi(x, r \cos \theta, r \sin \theta) d \theta
$$

we obtain

$$
\varphi^{0}(x, r)=a_{0}+a_{1} x+\frac{1}{4 \pi} \int_{\Gamma} b\left[\frac{\partial \varphi^{0}}{\partial n^{\prime}}\right] d \sigma \int_{0}^{2 \pi} \frac{d \theta}{\left[\left(x-x^{\prime}\right)^{2}+r^{2}+b^{2}-2 r b \cos \theta\right]^{t}}
$$

where now the outer integral is a line integral in a meridian plane along the open or closed curve $\Gamma$. We assume for the present that $\Gamma$ has a parametric 
representation which is $C^{\prime}$ in some interval. Similarly, if we put

$$
\varphi_{c}^{\prime}(x, r)=\frac{1}{\pi} \int_{0}^{2 \pi} \varphi(x, r \cos \theta, r \sin \theta) \cos \theta d \theta
$$

and

$$
\varphi_{s}^{\prime}(x, r)=\frac{1}{\pi} \int_{0}^{2 \pi} \varphi(x, r \cos \theta, r \sin \theta) \sin \theta d \theta,
$$

we obtain from equation (3.3)

$$
\varphi_{c}^{\prime}(x, r)=\frac{1}{4 \pi} \int_{\Gamma} b\left[\frac{\partial \varphi_{c}^{\prime}}{\partial n^{\prime}}\right] d \sigma \int_{0}^{2 \pi} \frac{\cos \theta d \theta}{\left[\left(x-x^{\prime}\right)^{2}+r^{2}+b^{2}-2 r b \cos \theta\right]^{\frac{3}{3}}}+a_{2} r
$$

and a second with the subscript $c$ replaced by $s$ and $a_{2}$ by $a_{3}$.

We have seen in $\S 2$ that the inner integrals in (3.4) and (3.5) may be replaced by (2.4) with proper weight factors. For example, (3.4) becomes either

$$
\varphi^{0}(x, r)=a_{0}+a_{1} x+\frac{1}{4 \pi} \int_{\Gamma} b\left[\frac{\partial \varphi^{0}}{\partial n^{\prime}}\right] d \sigma \int_{0}^{2 \pi} \frac{d \theta}{\left[\left(x-x^{\prime}+i r \cos \theta\right)^{2}+b^{2}\right]^{\frac{1}{2}}}
$$

or

$$
\varphi^{0}(x, r)=a_{0}+a_{1} x+\frac{1}{4 \pi} \int_{\Gamma} b\left[\frac{\partial \varphi^{0}}{\partial n^{\prime}}\right] d \sigma \int_{0}^{2 \pi} \frac{d \theta}{\left[\left(x-x^{\prime}+i b \cos \theta\right)^{2}+r^{2}\right]^{\frac{1}{t}}}
$$

Observe that the kernel has a weak singularity. (3.4a) is of some interest. If we note that $b$ and $x^{\prime}$ are functions of the parameter $\sigma$, we can, upon interchanging integrals, write (3.4a) as

$$
\begin{aligned}
\varphi^{0}(x, r) & =a_{0}+a_{1} x+\frac{1}{4 \pi} \int_{0}^{2 \pi} d \theta \int_{\Gamma} b\left[\frac{\partial \varphi^{0}}{\partial n^{\prime}}\right] \frac{d \sigma}{\left[\left(x-x^{\prime}+i r \cos \theta\right)^{2}+b^{2}\right]^{\frac{t}{2}}} \\
& =a_{0}+a_{1} x+\frac{1}{2 \pi} \int_{0}^{\pi} f(x+i r \cos \theta) d \theta
\end{aligned}
$$

since the integral over $\Gamma$ is merely a function of $x+i r \cos \theta$. The integral in (3.4c) is the Laplace representation for a solution of equation (1.2). When $(x, r)$ is a point on $\Gamma,(3.4 c)$ is actually an integral equation for $f(x)$, where

$$
f(x)=\int_{\Gamma} b\left[\frac{\partial \varphi^{0}}{\partial n^{\prime}}\right] \frac{d \sigma}{\left[\left(x-x^{\prime}\right)^{2}+b^{2}\right]^{\frac{1}{3}}}=2\left[\varphi^{0}(x, 0)-a_{0}-a_{1} x\right],
$$

thereby demonstrating that we can determine an axially symmetric potential by a knowledge of the potential on the axis of symmetry. We shall produce examples in which the use of both (2.3) and (2.4) will enable us to avoid the methods which have been previously employed by Heins and MacCamy ([2]) and Collins ([3]). 
The representation (3.5) now needs some comment. If we use (2.4), then it becomes

$$
\varphi_{c}^{\prime}(x, r)=a_{2} r+\frac{1}{2 \pi} \int_{\Gamma} r b^{2}\left[\frac{\partial \varphi_{c}^{\prime}}{\partial n^{\prime}}\right] d \sigma \int_{0}^{\pi} \frac{\sin ^{2} \theta d \theta}{\left[\left(x-x^{\prime}+i r \cos \theta\right)^{2}+b^{2}\right]^{t}} .
$$

Now $\varphi_{c}^{\prime}(x, r)$ may be written as $r A^{\prime}(x, r)$, where $A^{\prime}$ is a solution of equation (1.6) for $n=1$, and therefore

$$
A^{\prime}(x, r)=a_{2}+\frac{1}{2 \pi} \int_{\Gamma} b^{2}\left[\frac{\partial \varphi_{c}^{\prime}}{\partial n^{\prime}}\right] d \sigma \int_{0}^{\pi} \frac{\sin ^{2} \theta d \theta}{\left[\left(x-x^{\prime}+i r \cos \theta\right)^{2}+b^{2}\right]^{\frac{1}{2}}}
$$

If we are tempted to interchange the order of the integration, we do indeed have a representation for a solution to equation (1.2) provided that $(x, r)$ is not on $\Gamma$. This may be written as

$$
A^{\prime}(x, r)=a_{2}+\frac{1}{2 \pi} \int_{0}^{\pi} f_{1}(x+i r \cos \theta) \sin ^{2} \theta d \theta
$$

which is again a Laplace integral. However, because of the nature of the kernel which forms the representation (3.6a), that is,

$$
f_{1}(x+i r \cos \theta)=\int_{\Gamma} b^{2}\left[\frac{\partial \varphi_{c}^{\prime}}{\partial n^{\prime}}\right] \frac{d \sigma}{\left[\left(x-x^{\prime}+i r \cos \theta\right)^{2}+b^{2}\right]^{\frac{2}{3}}},
$$

we can no longer attempt to take the point $(x, r)$ on $\Gamma$ since now the kernel has no longer a weak singularity. All is not lost, however, for if we integrate the inner integral by parts, we can lower the degree of the singularity to obtain

$$
\begin{aligned}
A^{\prime}(x, r) & =a_{2}+\frac{1}{2 \pi r i} \int_{\Gamma}\left[\frac{\partial \varphi_{c}^{\prime}}{\partial n^{\prime}}\right] d \sigma \int_{0}^{\pi} \frac{\cos \theta\left(x-x^{\prime}+i r \cos \theta\right) d \theta}{\left[\left(x-x^{\prime}+i r \cos \theta\right)^{2}+b^{2}\right]^{\frac{t}{2}}} \\
& =a_{2}+\frac{1}{2 \pi r i} \int_{0}^{\pi} f_{2}(x+i r \cos \theta) \cos \theta d \theta
\end{aligned}
$$

The last integral in (3.6b) is no longer a Laplace integral. We shall not use this latter form in the examples which we shall present in the next section but shall try to take advantage of the two complex forms we have for the fundamental solution to produce a Volterra integral equation directly.

The situation for the Neumann problem is not as simple. From the Beltrami-Stokes equations, we have that (up to a weight factor) the normal derivative of solutions of equation (1.6) on the same curve is equal to the tangential derivative of the corresponding stream function. However, the classical Neumann problems of potential theory demand the vanishing of the normal derivative of $\varphi$ (a solution of equation (3.1)) on $\partial S$. In the case of the axially symmetric geometry which we discuss here, this means 
that the normal derivative of solutions of equation (3.1) is the normal derivative of solutions of equation (1.6) which have been weighted by a factor $r^{n}$ (the $n$ corresponding to the order of the harmonic). Hence, only in the case $n=0$ can we identify solutions of (1.6) and axially symmetric solutions of (3.1). In this case, the correspondence principle of Weinstein ([1]) may be employed, for then the vanishing of the normal derivative of a potential function is equivalent to the vanishing of the tangential derivative of the corresponding function. If the stream function $\psi$ is chosen to vanish on $\Gamma$, we find for the case when $n=0$ that it may be expressed as $r^{2} A^{\prime}$, so that we can reduce this case to a Dirichlet problem. We shall comment further on this at a later date.

\section{Some examples}

We now form integral equations for a specific geometry, making use of the two representations (3.4a) and (3.6b). $\Gamma$ is a surface of revolution generated by a circular arc drawn symmetrically with respect to the $x$-axis. We use (3.3) in two situations: (i) $a_{0}=1, a_{1}=a_{2}=a_{3}=0$ and (ii) $a_{0}=a_{1}=0, a_{2}=a_{3}=1$. If the points $\left(x^{\prime}, b\right)$ and $(x, r)$ are on $\Gamma$, the fundamental solution required for case (i) may be written as follows:

$$
\frac{1}{2 \pi} \int_{0}^{\pi} \frac{d \theta}{\left[\left(x-x^{\prime}+i r \cos \theta\right)^{2}+b^{2}\right]^{\frac{1}{2}}}=\frac{1}{2 \pi i} \int_{x-i r}^{x+i r} \frac{d t}{\left\{\left[(t-x)^{2}+r^{2}\right]\left[(t-a)^{2}+b^{2}\right]\right\}^{\sharp}} .
$$

On a circular arc of radius $a$, we may put $x=a \cos \alpha, r=a \sin \alpha$, and $x^{\prime}=a \cos \beta, \quad b=a \cos \beta,-\gamma \leqslant \alpha, \beta \leqslant \gamma$. Then with the substitution $t=a e^{i \lambda}$, we may express the last integral as

$$
\frac{1}{2 \pi a} \int_{-\alpha}^{\alpha} \frac{d \lambda}{[(\cos \lambda-\cos \alpha)(\cos \lambda-\cos \beta)]^{\frac{1}{2}}} .
$$

This integral is real if $\beta>\alpha$ and the question arises how we may define it for $\beta<\alpha$. Instead of confronting that problem, we give a second representation for the fundamental solution, of the same general form, and which is real for $\beta<\alpha$. We recall that the fundamental solution may also be written as

$$
\begin{aligned}
\frac{1}{2 \pi} \int_{0}^{\pi} \frac{d \theta}{\left[\left(x-x^{\prime}+i b \cos \theta\right)^{2}+y^{2}\right]^{\frac{1}{t}}} & =\frac{1}{2 \pi i} \int_{x^{\prime}-i b}^{x^{\prime}+i b} \frac{d t}{\left\{\left[(t-x)^{2}+y^{2}\right]\left[(t-a)^{2}+b^{2}\right]\right\}^{\frac{t}{t}}} \\
& =\frac{1}{2 \pi a} \int_{-\beta}^{\beta} \frac{d \lambda}{[(\cos \lambda-\cos \beta)(\cos \lambda-\cos \alpha)]^{\frac{1}{2}}},
\end{aligned}
$$

which is real for $\beta<\alpha$. 
Hence for case (i), we have for the boundary condition $\varphi^{0}(x, r)=0$ on $\Gamma$, the integral equation

$$
\begin{aligned}
1+\frac{1}{\pi} \int_{0}^{\alpha} b\left[\frac{\partial \varphi^{0}}{\partial n^{\prime}}\right] & d \beta \int_{0}^{\beta} \frac{d \lambda}{[(\cos \lambda-\cos \alpha)(\cos \lambda-\cos \beta)]^{3}} \\
& +\frac{1}{\pi} \int_{\alpha}^{\gamma} b\left[\frac{\partial \varphi^{0}}{\partial n^{\prime}}\right] d \beta \int_{0}^{\alpha} \frac{d \lambda}{[(\cos \lambda-\cos \alpha)(\cos \lambda-\cos \beta)]^{\frac{1}{t}}}=0 .
\end{aligned}
$$

This, in turn, may be rewritten as

$$
1+\frac{1}{\pi} \int_{0}^{\alpha} \frac{d \lambda}{(\cos \lambda-\cos \alpha)^{\frac{1}{2}}} \int_{\lambda}^{\gamma} b\left[\frac{\partial \varphi^{0}}{\partial n^{\prime}}\right] \frac{d \beta}{(\cos \lambda-\cos \beta)^{\frac{1}{2}}}=0 .
$$

Equation (4.1a) is a Volterra integral equation and indeed it may be converted to an Abel integral equation. We shall take advantage of this latter fact to solve (4.1a), multiply it by $\sin \alpha(\cos \alpha-\cos \rho)^{\sharp}$, and integrate from 0 to $\rho$. Then

$$
2 \sqrt{ }(1-\cos \rho)+\int_{0}^{\rho} d \lambda \int_{\lambda}^{\gamma} b\left[\frac{\partial \varphi^{0}}{\partial n^{\prime}}\right] \frac{d \beta}{(\cos \lambda-\cos \beta)^{\frac{1}{2}}}=0 .
$$

Upon differentiating with respect to $\rho$ and simplifying, we get

$$
\sqrt{2} \cos \frac{1}{2} \rho+\int_{\rho}^{\gamma} b\left[\frac{\partial \varphi^{0}}{\partial n^{\prime}}\right] \frac{d \beta}{(\cos \rho-\cos \beta)^{\frac{1}{t}}}=0 .
$$

This last equation is still of the Abel type, and if we repeat the previous step we get

$$
\begin{aligned}
\pi \int_{\rho}^{\gamma} b\left[\frac{\partial \varphi^{0}}{\partial n}\right] d \beta+2 \cos ^{2} \frac{1}{2} \rho & \left(\frac{\pi}{2}-\arcsin \frac{\cos \frac{1}{2} \gamma}{\cos \frac{1}{2} \rho}\right) \\
& +2 \cos \frac{1}{2} \gamma\left(\cos ^{2} \frac{1}{2} \rho-\cos ^{2} \frac{1}{2} \gamma\right)^{\frac{1}{2}}=0, \quad 0 \leqslant \rho \leqslant \gamma .
\end{aligned}
$$

Hence, we may either calculate $\left[\partial \varphi^{0} / \partial n\right]$ on the bowl (for the case in which the potential at infinity is unity) by differentiating with respect to $\rho$ or we may determine the total change on this spherical bowl by evaluating

$$
2 \pi a^{2} \int_{0}^{\gamma} \sin \beta\left[\frac{\partial \varphi^{0}}{\partial n^{\prime}}\right] d \beta=2 \pi a \int_{0}^{\gamma} b\left[\frac{\partial \varphi^{0}}{\partial n^{\prime}}\right] d \beta .
$$

The second example (ii) that we discuss involves the integral representation (3.5a) and we recall that the inner integral may be replaced by an integral of the same form with $\left(x^{\prime}, b\right)$ replaced by $(x, r)$ as well as $(x, r)$ replaced by $\left(x^{\prime}, b\right)$. Then we have for $\left(x^{\prime}, b\right)$ and $(x, r)$ on $\Gamma$,

$$
\int_{0}^{\pi} \frac{\sin ^{2} \theta d \theta}{\left[\left(x-x^{\prime}+i r \cos \theta\right)^{2}+b^{2}\right]^{\frac{3}{3}}}=\frac{a}{2 b^{2} r^{2}} \int_{-\alpha}^{\alpha} \frac{\left(e^{i \lambda}-\cos \alpha\right)\left(e^{i \lambda}-\cos \beta\right) d \lambda}{[(\cos \lambda-\cos \alpha)(\cos \lambda-\cos \beta)]^{3}}
$$


Now since the denominator of this last integral is real for $\beta>\alpha$, we may write it as

$$
-\frac{a}{b^{2} r^{2}} \int_{0}^{\alpha}\left\{[(\cos \lambda-\cos \alpha)(\cos \lambda-\cos \beta)]^{\frac{1}{}}-\frac{\sin ^{2} \lambda}{[(\cos \lambda-\cos \alpha)(\cos \lambda-\cos \beta)]^{3}}\right\} d \lambda
$$

in this case. Similarly, we may write the right-hand member of (4.2) for $\beta<\alpha$ as

$$
-\frac{a}{b^{2} r^{2}} \int_{0}^{\beta}\left\{[(\cos \lambda-\cos \alpha)(\cos \lambda-\cos \beta)]^{\downarrow}-\frac{\sin ^{2} \lambda}{[(\cos \lambda-\cos \alpha)(\cos \lambda-\cos \beta)]^{1}}\right\} d \lambda .
$$

Following our discussion in problem (i) of this section, we find that the vanishing of $\varphi^{\prime}$ on $\Gamma$ is equivalent to the integral equation

$$
\begin{aligned}
\frac{1}{2 \pi} \int_{0}^{\alpha} d \lambda \int_{\lambda}^{\gamma}\left[\frac{\partial \varphi^{1}}{\partial n^{\prime}}\right] d \beta\left\{[(\cos \lambda-\cos \alpha)(\cos \lambda-\cos \beta)]^{\dagger}\right. \\
\left.-\frac{\sin ^{2} \lambda}{[(\cos \lambda-\cos \alpha)(\cos \lambda-\cos \beta)]^{\frac{1}{t}}}\right\} d \lambda=a \sin ^{2} \alpha .
\end{aligned}
$$

The solution of this integral equation is not without interest. We make use of the same device that was employed in problem (i). We multiply equation (4.3) by $\sin \alpha /(\cos \alpha-\cos \rho)^{\frac{1}{2}}$ and integrate from 0 to $\rho$. Then (4.3) becomes

$$
\begin{gathered}
(16 a \sqrt{2} / 15)\left[5 \sin ^{3} \frac{1}{2} \rho-4\right. \\
\left.=\frac{1}{4} \int_{0}^{\frac{5}{2}} \frac{1}{2} \rho\right] \\
\quad-\frac{1}{2} \int_{0}^{\rho} d \lambda \int_{\lambda}^{\gamma} \frac{\sin ^{2} \lambda}{(\cos \lambda-\cos \beta)^{\frac{1}{3}}}\left[\frac{\partial \varphi^{1}}{\partial n^{\prime}}\right] d \beta .
\end{gathered}
$$

If we differentiate this equation with respect to $\rho$ we obtain

$$
\begin{aligned}
(4 a \sqrt{2} / 3) \sin \frac{3}{2} \rho=\frac{1}{4} \int_{0}^{\rho} d \lambda \int_{\lambda}^{\gamma}(\cos \lambda-\cos \beta)^{\frac{1}{2}}\left[\frac{\partial \varphi^{1}}{\partial n^{\prime}}\right] d \beta \\
\quad-\int_{\rho}^{\gamma} \frac{\sin \rho}{(\cos \rho-\cos \beta)^{\frac{1}{1}}}\left[\frac{\partial \varphi^{1}}{\partial n^{\prime}}\right] d \beta .
\end{aligned}
$$

Hence (4.4) becomes an integro-differential equation if we put

$$
\int_{\lambda}^{\gamma}(\cos \lambda-\cos \beta)^{\frac{\beta}{[}}\left[\frac{\partial \varphi^{1}}{\partial n^{\prime}}\right] d \beta=h(\lambda)
$$

that is,

$$
\frac{1}{4} \int_{0}^{\rho} h(\lambda) d \lambda+h^{\prime}(\rho)=\frac{1}{3} \sqrt{ } 2 \sin \frac{3}{2} \rho
$$


This equation has a solution

$$
h(\lambda)=A \cos \frac{1}{2} \lambda-\sqrt{ } 2 \cos \frac{3}{2} \lambda,
$$

where $A$ is an arbitrary constant. The arbitrary constant indicates that the homogeneous part of equation (4.4) has a non-trivial solution and the question now remains how to evaluate the constant $A$. We do not have to go far to find a method. If we examine the integral equation (4.5) (which is now of the same form which we encountered in problem (i)), we find that

$$
\begin{aligned}
\frac{\pi}{2} \int_{\rho}^{\gamma}\left[\frac{\partial \varphi^{1}}{\partial n^{\prime}}\right](\cos \rho-\cos \beta) d \beta= & 2(\cos \rho-\cos \gamma)^{\frac{t}{}}\left(A \cos \frac{1}{2} \gamma-\sqrt{2} \cos \frac{3}{2} \gamma\right) \\
& +\int_{\rho}^{\gamma}(\cos \rho-\cos \lambda)^{\frac{1}{t}}\left(A \sin \frac{1}{2} \lambda-3 \sqrt{ } 2 \sin \frac{3}{2} \lambda\right) d \lambda
\end{aligned}
$$

or

where

$$
\begin{aligned}
\frac{\pi}{2} \int_{\rho}^{\gamma}\left[\frac{\partial \varphi^{1}}{\partial n^{\prime}}\right] d \beta=\frac{A \cos \frac{1}{2} \gamma-\sqrt{ } 2 \cos \frac{3}{2} \gamma}{(\cos \rho-\cos \gamma)^{\frac{1}{2}}} \\
+\frac{1}{2} \sqrt{ } 2\left[(A+3 \sqrt{ } 2)\left(\frac{1}{2} \pi-\Phi_{1}\right)-6 \sqrt{ } 2 \cos ^{2} \frac{1}{2} \rho\left(\frac{1}{2} \pi-\Phi_{1}\right)\right. \\
\left.-6 \sqrt{ } 2 \cos \frac{1}{2} \gamma\left(\cos ^{2} \frac{1}{2} \rho-\cos ^{2} \frac{1}{2} \gamma\right)^{\frac{1}{1}}\right],
\end{aligned}
$$

$$
\Phi_{1}=\arcsin \frac{\cos \frac{1}{2} \gamma}{\cos \frac{1}{2} \rho} .
$$

But (4.6) would imply that $\left[\partial \varphi^{1} / \partial n\right]$ would have a non-integrable singularity at $\beta=\alpha$ and this can only be avoided by taking $A \cos \frac{1}{2} \gamma-\sqrt{2} \cos \frac{3}{2} \gamma=0$. Hence

$$
\begin{array}{r}
{\left[\frac{\partial \varphi^{1}}{\partial n^{\prime}}\right]=\frac{2}{\pi}\left[\frac{3 \cos \frac{1}{2} \gamma \sin \rho}{\left(\cos ^{2} \frac{1}{2} \rho-\cos ^{2} \frac{1}{2} \gamma\right)^{\frac{1}{2}}}+3 \sin \rho\left(\frac{1}{2} \pi-\Phi_{1}\right)\right.} \\
\left.-\frac{2 \cos ^{3} \frac{1}{2} \gamma \sin \rho}{(1+\cos \rho)\left(\cos ^{2} \frac{1}{2} \rho-\cos ^{2} \frac{1}{2} \gamma\right)^{\frac{1}{3}}}\right] .
\end{array}
$$

The use of the transformed fundamental solution (2.3) and (2.4) gives us considerably more latitude than if use is made of the classical formulation of Fredholm. For one thing, we can derive many of the integral equations which have appeared in the past two decades and which have often been given as an assumption, subject to verification. We are now aware that these integral equations are transformed versions of the Fredholm integral equations which normally arise in the formulation of boundary-value problems of potential theory. Example (ii) of this section shows us that we can consider the higher harmonics about the $z$-axis. 


\section{AXIALLY SYMMETRIC POTENTIAL THEORY}

Hence we can formulate Volterra integral equations which contain information about polarization coefficients as well as some information about virtual mass coefficients ([8]). This topic has not received much attention in axially symmetric potential theory. Indeed the term 'axially symmetric' is often taken to mean a study of boundary-value problems for axially symmetric geometries with axially symmetric boundary conditions of infinity.

\section{REFERENCES}

1. A. Weinstein, 'Discontinuous integrals and generalized potential theory', Trans. Amer. Math. Soc. 63 (1948) 342-54.

2. A. E. Herns, 'Axially symmetric boundary-value problems', Bull. Amer. Math. Soc. 71 (1965) 787-808.

3. W. D. Collins, 'On the solutions of some axisymmetric boundary value problems by means of definite integrals, I. Some electrostatic and hydrodynamic problems for a spherical cap.' Quart. J. Mech. Appl. Math. 12 (1959) 232-41.

4. W. H. Fleming, Functions of several variables (Addison-Wesley, Reading, Mass. 1965), pp. 162, 183.

5. G. N. Watson, $A$ treatise on the theory of Bessel functions, 2nd edn (Cambridge University Press, 1944), p. 384.

6. S. D. Porsson, 'Sur l'intégration des équations linéares aux différences partielles', J. École Roy. Polytéchnique 12 (1923) 215-48.

7. O. D. KellogG, Foundations of potential theory (Springer, Berlin, 1929), p. 105.

8. M. Schiffer and G. Szeqö, 'Virtual mass and polarization', Trans. Amer. Math. Soc. 67 (1948) 130-205.

Department of Mathematics

University of Michigan

Ann Arbor

Michigan 48104 